\title{
EFECTO GENOTOXICO DEL AGUA RESIDUAL DE LA CURTIEMBRE SAN FAUSTINO-NORTE DE SANTANDER-COLOMBIA
}

\section{GENOTOXIC EFFECT OF WASTEWATER FROM THE SAN FAUSTINO- NORTE DE SANTANDER-COLOMBIA TANNERY}

\author{
Esp. Quintero Salamanca Greila Paola *, Phd. Quijano Parra Alfonso**, \\ Mg. Melendez Gelvez Ivan *,**
}

*Universidad de Pamplona, Facultad de Ciencias Básicas, Departamento de BiologíaQuímica. caritotolosa@gmail.com.

** Universidad de Pamplona. Profesor titular. Departamento de Química y Biología. Facultad de Ciencias Básicas. Grupo de Investigación en Química; alfonsoquijanoparra@gmail.com. Grupo de Investigación en Química. Laboratorio de Control de Calidad.

***Universidad de Pamplona. Profesor asociado. Departamento de Química-Biología. Facultad de Ciencias Básicas.imgelvez@unipamplona.edu.co. Grupo de Investigación en Biología Molecular

Resumen: Las industrias curtiembres impactan nocivamente el ecosistema por las considerables cantidades de residuos orgánicos y tóxicos que generan, tales como sales y metales pesados; siendo estos últimos, altamente tóxicos, según la IARC (International Agency for Research on Cancer) por inducir efectos mutagénicos y genotóxicos en los seres humanos.

La problemática se presenta por el mal manejo de los desechos, debido a la falta de cultura en la preservación y cuidado de los recursos naturales, la desinformación y la no concientización de las secuelas que se generan, siendo una de las más relevantes que este recurso hídrico contaminado puede llegar a ser usado indiscriminadamente por la población; por tal motivo se hace necesario estimar el impacto ambiental generado. La importancia de este estudio radica en que los desechos de curtiembres ubicadas en la ciudad de Cúcuta, pueden llegar al río Pamplonita, y contaminarlo con metales pesados, como lo reportan estudios realizados en las aguas de desecho de la curtiembre Tasajero. En este estudio se identificaron los metales pesados $(\mathrm{Cr}, \mathrm{Pb}, \mathrm{Cd}, \mathrm{Fe}, \mathrm{Zn}, \mathrm{Cu})$ presentes en el agua residual proveniente de la curtiembre San Faustino (Cúcuta), utilizando la técnica de espectroscopia de absorción atómica; encontrándose concentraciones perjudiciales para el ser humano. La mutagenicidad se determninó a través del test de Ames, con las cepas de Salmonella typhimurium TA98 y TA100. Para determinar la genotoxicidad, se usó el ensayo cometa. Se encontró que las aguas de desecho de la curtiembre San Faustino, inducen un incremento en la mutagenicidad en las cepas TA98 y TA100 de Salmonella typhimurium. De igual manera, se encontró que las aguas de desecho de la curtiembre San Faustino, provocan daño al ADN en linfocitos humanos.

Palabras clave: Salmonella typhimurium TA-98, TA-100, metales pesados, mutagenicidad, genotoxicidad, curtiembre. 


\begin{abstract}
The leather tannining industries impacts harmfully the ecosystem by the considerable amounts of organic and toxic waste they generate, such as salts and heavy metals; being the last ones, highly toxic, according to the IARC (international agency for researching on cancer) because it induces mutagenic and genotoxic effects in humans.
\end{abstract}

The problem is presented by the poor management of waste, in addition to the lack of culture in the preservation and the environmental care, the lack of information and not being aware of the consequences that are generated, being one of the most relevant this contaminated water resource can be used indiscriminately by the population; for this reason it is necessary to estimate the environmental impact generated. The importance of this study lies on the possibility of heavy metals arriving to the Pamplonita River, and contaminate it, due the tanneries wastes from Cúcuta, as the results made over wastewater of Tasajero tannery show.

In this study we identified the heavy metals $(\mathrm{Cr}, \mathrm{Pb}, \mathrm{Cd}, \mathrm{Fe}, \mathrm{Zn}, \mathrm{Cu})$ present in the wastewater from san faustino leather tannining industries y Cúcuta city, using the atomic absorption spectroscopy technique; concentrations are found to be harmful to humans. The mutagenicity ws detected through the Ames test, with the strains of Salmonella typhimurium TA98 and TA100. To determine genotoxicity, the comet assay was used. It was found that the wastewater from the San Faustino tannery induces an increase in mutagenicity in the TA98 and TA100 strains of Salmonella typhimurium. Equally, it was found that wastewater from San Faustino tannery causes DNA damage in human lymphocytes.

Keywords: Salmonella typhimurium TA- 98, TA-100, heavy metals, genotoxicity , tannery..

\section{INTRODUCCIÓN}

El río Pamplonita recorre gran parte de los municipios del departamento Norte de Santander; como son, Pamplona, Pamplonita, Bochalema, Chinácota, Herrán, Ragonvalia, Los Patios, Cúcuta, Villa del Rosario y Puerto Santander;1 a su vez, estos municipios utilizan dicha fuente hídrica para múltiples fines, tales como agricultura, comercio y uso doméstico; lo cual se evidencia principalmente en la zona metropolitana de Cúcuta; sin embargo, éste afluente presenta una grave problemática medio ambiental y sanitaria debido a que es receptor de los desechos no sólo orgánicos, sino también industriales; 2 de los municipios en cuestión, incidiendo directamente en la calidad de vida de su población y causando efectos ambientales como la disminución de la biodiversidad acuática.

Dentro de los residuos industriales, se encuentran los vertimientos de curtiembres situadas a lo largo del río; estas empresas, desarrollan la adecuación de pieles, mediante la modificación de su aspecto químico y físico, en el cual se estabiliza el colágeno de la piel mediante el uso de sustancias como el ácido fórmico y el cromo, este método conocido como proceso de curtido, está destinado a producir tales transformaciones en las pieles, y sean convertidas en un material duradero, casi imputrescible, apenas permeable al agua y a la vez suave, elástico y flexible, cuyo producto final es el cuero; 3,4 orientando su actividad productiva hacia el marketing de productos de marroquinería, talabartería y calzado.

Los residuos de metales pesados en los hábitats contaminados 5 pueden acumularse en microorganismos, flora6 y fauna acuática,7 los cuales, a su vez, puede entrar en la cadena alimenticia humana y dar lugar a problemas de salud.,8,9,10. La ingesta produce un cuadro gastrointestinal en forma de vómitos, dolores abdominales, diarreas y hemorragias intestinales11. Se han descrito casos de insuficiencia renal aguda12 del hígado, de la glándula tiroides y la 
médula ósea y hasta la muerte, por colapso cardiocirculatorio.

Numerosos estudios han demostrado la presencia del $\mathrm{Cr}+6$, uno de estos metales pesados, en los vertimientos de curtiembres5, 13, 14, 15, 16, 17; para este contaminante la concentración máxima permisible es de $0,5 \mathrm{mg} / \mathrm{L}$, en agua residuales de curtiembre.15 Este compuesto hexavalente se absorbe por vía cutánea y respiratoria; penetra con facilidad en el interior de los eritrocitos. La vía de absorción cutánea es de más fácil acceso al organismo, esta exposición produce afecciones locales sobre la piel causando dermatitis, sensibilización de la piel y mucosas; y por exposición a la vía respiratoria, produce afecciones generales como tos, bronquitis crónica, ulceraciones del tabique nasal, dolores respiratorios y de cabeza, hemorragia nasal18 .

Debido a que la calidad del cuero depende principalmente de la cantidad y homogeneidad del cromo fijado en el colágeno de la piel, el proceso de curtido utiliza exceso de cromo para garantizar la fijación de éste en las proteínas de la piel y evitar la descomposición del cuero19. Sin embargo; hay que tener en cuenta que el cromo se encuentra en varios estados de oxidación, siendo los más comunes, el $\mathrm{Cr}+3$ y el $\mathrm{Cr}+6$; el primero es imprescindible para el ser humano y el último, se comporta como un elemento altamente nocivo.20, 21, 22, 23. El $\mathrm{Cr}+3$ es un oligoelemento, indispensable para procesos bioquímicos y fisiológicos necesarios para la vida. $\mathrm{El} \mathrm{Cr}+6$ presenta una elevada toxicidad, alta persistencia y rápida acumulación por los organismos vivos, sus efectos no se detectan fácilmente a corto plazo; y difiere de otros contaminantes por no ser biodegradable24 y bioacumularse.25,7. Este elemento, al ingresar a los organismos, ya sea por ingestión, contacto o inhalación, genera efectos muy nocivos de orden genético, mutagénico y carcinógeno26. Encontrándose en la clasificación de la IARC (International Agency for Research on Cancer) en el grupo I (cancerígeno comprobado en humanos)27, ya que en contacto directo produce cáncer; conduce a la replicación disfuncional del ADN, desregulación de sitios de control del ciclo celular, desregulación de sistemas de reparación, inestabilidad de microsatélites, respuesta inflamatoria y el daño de genes, clave en la regulación de redes responsables del balance, entre la vida y la muerte celular.

Actualmente es muy discutido el riesgo de ingestión del $\mathrm{Cr}$ (VI) en aguas de consumo.
Estudios in vivo han mostrado que el Cr (VI) es un genotóxico marcadamente dependiente de la dosis con la evidencia de un fuerte efecto umbral, debido a la desintoxicación extracelular (por reducción a Cr (III)) antes de la absorción por órganos y tejidos periféricos28. Estudios en ratas y ratones muestran aberraciones cromosómicas en médula ósea, ruptura de cadena simple en ADN de hígado y cerebro, como también ruptura de cadena simple de ADN en leucocitos29,30. Ciertas formas de cromo hexavalente son conocidas como carcinógenos respiratorios que inducen un amplio espectro de daños en el ADN31, 32. Lesiones genéticas estructurales producidas por el $\mathrm{Cr}(\mathrm{VI})$ inducen aductos, rupturas, uniones proteína-ADN, oxidación de bases, sitios AP y entrecruzamientos entre cromátidas33,34 . La asociación del cromo con el enlace fosfodiester, es una de las lesiones más abundante inducidas por este en células de mamífero y se piensa que es la primera causa de mutagenicidad32. El daño inducido por cromo conduce a la replicación disfuncional del ADN, causando sustitución y delección de bases, que son sustrato para los sistemas de reparación por escisión de bases (BER) y reparación por escisión de nucleótidos (NER), promoviendo así la inestabilidad genómica35.

Hay varias líneas de evidencia que sugieren la importancia de la sustitución de bases en la mutagenicidad del cromo, siendo la sustitución G: $\mathrm{C}$ el blanco primario tanto in vivo como in vitro36. La reducción del $\mathrm{Cr}(\mathrm{VI})$ ha sido mostrada para producir especies reactivas tales como radicales libres, anión superoxido y radicales hidroxil, posiblemente a través de la reacciones del $\mathrm{Cr}(\mathrm{V})$ y $\mathrm{Cr}(\mathrm{IV})$ con el peróxido de hidrogeno similares a la reacción de Fenton29,37. .Además se ha encontrado que en presencia de vitamin $\mathrm{A}$, en celulas V79 se incrementa el radical hidroxilo, las aberracciones cromosómicas y las mutaciones sobre el locus HGPRT38,39. El daño inducido por el cromo (VI) conduce a la replicación disfuncional del ADN, desregulación de sitios de control del ciclo celular, desregulación de sistemas de reparación, inestabilidad de microsatélites, respuesta inflamatoria y el daño de genes clave en la regulación de redes responsables del balance entre la vida y la muerte celular, los cuales juegan un papel importante en la carcinogénesis del cromo (VI). Varias líneas de evidencia indican que la progresión neoplásica es el resultado de cambios epigenéticos y genéticos que confieren a la célula ventajas de supervivencia, que en último término conducen a la conversión de una célula normal a una célula maligna.La vía de absorción cutánea es 
la de más fácil acceso al organismo, frente a lo cual cabe destacar el cuadro patológico de la dermatitis alérgica de contacto. En esta los compuestos de $\mathrm{Cr}$ $6+$ penetran en la piel más rápidamente que los compuestos de $\mathrm{Cr} 3+40$.

El objetivo de la presente investigación fue determinar la mutagenícidad y genotoxicidad en aguas residuales de curtiembres en la ciudad de Cúcuta, Norte de Santander. Para la determinación de la genotoxicidad, se utilizó el ensayo cometa que es una técnica altamente sensible para evaluar el daño y la reparación del ADN en cualquier tipo de célula eucariota. Para la determinación de la mutagenicidad se empleó el test de Ames, con las cepas de S. typhimurium TA-98 y TA-100

\section{METODOLOGİA}

\subsection{Toma de muestra}

Las muestras de agua se recolectaron en un botellón de polietileno previamente lavado, en una cantidad aproximada de 20 litros. Las muestras se obtuvieron antes de la curtiembre, dentro de la planta y en los vertimientos realizados después del proceso de desencalado, en la curtiembre San Faustino de la ciudad de Cúcuta, Norte de Santander.

\subsection{Concentración de la muestra de agua residual}

Las muestras de agua se concentraron a través de una columna que contenía $100 \mathrm{~g}$ de resina Amberlite XAD-2 a una velocidad de $15 \mathrm{ml} / \mathrm{min}$; después de pasar toda la muestra, la columna se eluyó con diclorometano. Posteriormente la muestra se concentró en un rotaevaporador a una temperatura de $60{ }^{\circ} \mathrm{C}$, a $150 \mathrm{rpm}$; hasta llevarla a sequedad. Seguidamente se diluyo el residuo en 20 ml de Dimetilsulfoxido al $3 \%$ para conservarla.

\subsection{Cuantificación de metales pesados.}

Para la determinación de metales presentes en los vertimientos de curtiembres, se utilizó la técnica de espectroscopia de absorción atómica (EAA) 41. Se utilizó una lámpara específica para cada metal en estudio ( $\mathrm{Cr}, \mathrm{Pb}, \mathrm{Cd}, \mathrm{Fe}, \mathrm{Zn}, \mathrm{Cu}$ ), desarrollando una curva de calibración utilizando disoluciones de concentración conocida preparadas a partir del estándar 1000 ppm.

\subsection{Detección de la actividad mutagénica}

El efecto mutagénico de los extractos de las aguas residuales de la curtiembre, se determinó por medio del test de Ames usando el protocolo descrito por 42. En esta prueba el indicativo de la mutación es la reversión de his- a his+, los revertantes se conocen porque crecen en medio mínimo sin histidina. Se trabajó con dos cepas de Salmonella thypimurium, la cepa TA 98 en la que se produce la reversión a his+ por ganancia en un par de bases en el gen mutado y la cepa TA 100 en la cual se produce la reversión por sustitución de un par de bases. Con cada muestra se trataron 107 bacterias, en ausencia de enzimas microsomales. Se utilizaron controles positivo y negativo. Para el control positivo se utilizó 4 -nitroquinolina oxido (4-NQO) y como control negativo se usó dimetilsulfoxido (DMSO) al $12 \%$. Se consideraron positivos los resultados que presentaron de forma reproducible, un incremento significativo de la mutación con respecto al control negativo. Para verificar la reproducibilidad de los resultados se hicieron tres experimentos independientes, cada uno por duplicado.

\subsection{Detección del daño del ADN}

Para detectar la ruptura del ADN, se utilizó el ensayo cometa. Se siguió la metodología propuesta por43 y modificada por44. La ocurrencia de daño en el ADN se basó en la longitud de la cola del cometa, inducida por la ruptura del ADN. Se consideró que la cola era producida por ruptura si era mayor de $26 \mu \mathrm{m}$ que es la longitud producida por factores espontáneos.

\subsection{Extracción de linfocitos}

Para los experimentos de genotoxicidad se usaron linfocitos de sangre periférica, los cuales se extrajeron de una persona joven y sana. Los linfocitos se separaron de la sangre total usando un gradiente de ficoll-hipaque. Seguidamente se determinó la viabilidad, la cual siempre se mantuvo por encima del $95 \%$. Tratamiento

Se tomaron $200 \mu l$ de células, las cuales se mezclaron con $50 \mu \mathrm{l}$ del tratamiento o control, posteriormente se incubó durante $1 \mathrm{~h}$ a $37^{\circ} \mathrm{C}$, luego se tomó $75 \mu$ l agarosa de bajo punto de fusión y se mezcló con $10 \mu \mathrm{l}$ de células tratadas para luego ser vertidas en una lámina base de agarosa de punto de fusión normal, luego se llevó a incubar $6 \mathrm{~min}$ a $4^{\circ} \mathrm{C}$. Después se retiró el cubre objeto y se adicionó otros $75 \mu \mathrm{l}$ de agarosa e incubó $6 \min$ a $4^{\circ} \mathrm{C}$. Terminada la incubación las láminas base se 
sumergieron en solución de lisis mínimo $1 \mathrm{~h}$. Posteriormente se lavaron las placas con PBS y se procedió a realizar la electroforesis, 1/2 hora sin conectar a la fuente de poder y media hora conectada a 25 v y300 mA; luego las placas se lavaron con solución neutralizante. Una vez terminado el proceso se procedió a la coloración con bromuro de etidio y a la observación en el microscopio de fluorescencia Olympus U-RFKT50 con el objetivo de $25 \mathrm{X}$. Se analizaron un total de 200 células

\section{RESULTADOS Y DISCUSIÓN}

Tabla 1: Concentraciones en partes por millón (ppm) de metales pesados permitidos en la norma de la OMS y las encontradas en los diferentes sitios de muestreo

\begin{tabular}{lllll}
\hline $\begin{array}{c}\text { Metal } \\
\text { Pesado }\end{array}$ & $\begin{array}{c}\text { Valores } \\
\text { máximos } \\
\text { permitidos }\end{array}$ & $\begin{array}{c}\text { Punto } \\
\text { Inicial }\end{array}$ & Curtiembre & $\begin{array}{c}\text { Punto } \\
\text { final }\end{array}$ \\
\hline $\mathbf{C r}$ & $\mathbf{0 . 0 5}$ & 0,2438 & 14.0087 & 0,4563 \\
$\mathbf{P b}$ & $\mathbf{0 . 0 5}$ & 0.3589 & 0.2929 & 0.6587 \\
$\mathbf{C d}$ & $\mathbf{0 . 0 0 5}$ & 0.0001 & 0.0002 & 0.0002 \\
$\mathrm{Fe}$ & $\mathbf{2}$ & $\mathrm{ND}$ & 8.6570 & 0.0835 \\
$\mathrm{Zn}$ & $\mathbf{1 5}$ & 0.1739 & 2.5690 & 0.2353 \\
$\mathbf{C u}$ & $\mathbf{2}$ & 0.0217 & 0.0849 & 0.232 \\
& & & & \\
\hline
\end{tabular}

Al comparar los valores obtenidos de metales con los límites máximos permisibles establecidos por la Organización Mundial de la Salud (OMS)45, los metales Cr, Pd; sobrepasan dichos límites en las aguas del río Pamplonita. Como se observa en la tabla. Por lo que el agua de este río representa riesgo para la población y el medio ambiente, ya que puede considerarse como altamente contaminado por Cobre de 5 a 9 veces mayor, comparados con en el nivel máximo permitido y por el plomo el cual sobrepasa los límites hasta 13 veces el valor. Las concentraciones de metales pesados antes mencionados, es atribuida a las descargas residuales de las curtiembres; debido a los desechos que en ellas se generan.

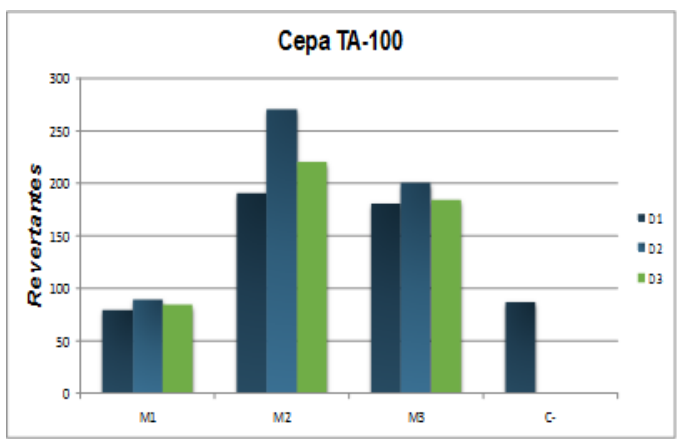

Fig. 1. Mutagenicidad inducida en Salmonella typhimurium TA-98 y TA-00 por extractos de aguas residuales de la curtiembre San Faustino de la ciudad de Cúcuta

De acuerdo a las revertantes observadas en la figura 1, se considera que la muestra es positiva para la cepa TA-98 ya que existe una relación de dosis de respuesta entre las concentraciones probadas y el número de revertantes inducidos; esto se puede determinar utilizando los criterios descritos en 46,47 donde la muestra se considera mutagénica cuando el número de colonias revertantes es $\geq 2$. La mutagenicidad observada la podemos atribuir a la presencia de metales pesados tales como $\mathrm{Cr}$ y $\mathrm{Hg}$ que son muy comunes en las aguas de residuo de las curtiembres según lo plantea47 . En el caso de la cepa TA-98 se observa que cada una de las dosis supera entre 3-4 veces el valor del control negativo, mientras que en la cepa TA-100 solamente observamos un incremento significativo de la mutagenicidad en las aguas dentro de curtiembre, lo cual nos indica que los compuestos vertidos por esta industria inducen mutación por pérdida o ganancia de bases y por sustitución de bases.

\section{Tabla 2: Daño del ADN en la sangre periférica humana (linfocitos) inducida por el extracto orgánico del agua de curtiembres}

\begin{tabular}{cc}
\hline Dosis $(\mu \mathrm{g})$ & $\begin{array}{c}\text { X promedio } \\
(\mu \mathrm{m})\end{array}$ \\
\hline $\mathrm{D} 1$ & 57 \\
$\mathrm{D} 2$ & 83 \\
$\mathrm{D} 3$ & 95 \\
$\mathrm{PBS}$ & 20 \\
$\mathrm{H}_{2} \mathrm{O}_{2} 50$ & 120 \\
$\mathrm{mM}$ & \\
\hline
\end{tabular}


Como control positivo se usó peróxido de hidrógeno (H2O2 $50 \mathrm{mM}$ ), como control negativo se utilizó PBS. Se determinó la longitud promedio de cola de un total de 200 células

Mediante el ensayo cometa, se encontró que las dosis ensayadas aportan daño al ADN en linfocitos humanos expuestos a los compuestos presentes en las aguas residuales de curtiembres, probablemente debido a la presencia de metales como $\mathrm{Cr}, \mathrm{Hg}$ y $\mathrm{Pb}$ esto se añade a la evidencia reportada por48, en relación con la sensibilidad de las cepas TA98, TA100, en el bioensayo de micro suspensión de Salmonella para detectar la actividad mutagénica de cromo (VI).

Los resultados los podemos explicar teniendo en cuenta que se ha demostrado que la carcinogenicidad del cromo puede ser promovida a través de varios mecanismos, incluyendo la reducción metabólica intracelular del $(\mathrm{Cr} \mathrm{VI})$, produciendo especies de cromo capaces de interactuar con el ADN, las cuales son las responsables de los efectos mutagénicos y genotóxicos29,49. El cromo también induce aberracciones cromosómicas, rupturas de cadena simple, aductos, sitios AP, oxidación de bases, entrecruzamientos, daño al enlace fosfodiester, daño en los sitemas de reparación, sustitución y delección de bases29,32,35. El daño encontrado, lo podemos atribuir, ademas además a la presencia de metales como el hierro, manganeso, plomo, cobre, zinc y níquel debido a su alta actividad mutagénica según fue identificado por46. Se observó una tendencia creciente de daño celular según el aumento de la concentración, aumentando el daño de manera progresiva en las diferentes concentraciones evaluadas

\section{CONCLUSIONES}

Las industria de curtiembres de la ciudad de Cucuta, están aportando a las aguas compuestos que inducen mutagenicidad en las cepas de S. typhimurium TA-98, dicha mutagenicidad, la podemos atribuir a la presencia de compuestos mutagénicos como metales pesados que pueden causar desplazamiento del marco de lectura o pérdida o ganancia de bases.

También detectamos que estas aguas residuales de curtiembres de Cúcuta inducen daño en el material genético.
Conflicto de intereses: El manuscrito fue preparado y revisado con la participación de todos los autores, quienes declaramos que no existe ningún conflicto de intereses que ponga en riesgo la validez de los resultados presentados

\section{REFERENCIAS}

ACHARYA,S; $\quad$ MEHTA,K;KRISHNAN,S; RAO,C.V.2001. A subtoxic interactive toxicity study of ethanol and chromium in male Wistar rats. Alcohol 23:99-108.

Alfonso Quijano Parra, Carol Castillo T, Iván. Meléndez G. Potencial Mutagénico $Y$ Genotóxico de Aguas Residuales de La Curtiembre Tasajero En La Ciudad de Cúcuta, Norte de Santander, Colombia. U.D.C.A Actual. Divulg. Científica 2015, 18, N.1, 13-20.

AMES, B.N;LEE, F.D;DURSTON, W.E.1973.An improved bacterial test system for the detection and classification of mutagens and carcinogens. Proc.Natl.Acad.Sci USA.70(3):782-6.

Baldrian, P. Interactions of Heavy Metals with White-Rot Fungi. Enzyme Microb. Technol. 2003, 32, 78-91.

Barba-Ho, Luz Edith; Ballesteros, Yojana V.; Patiño, Paola J.; Ramírez Callejas, C. IMPACTO GENERADO POR LOS VERTIMIENTOS DE LAS CURTIEMBRES EN CORRIENTES SUPERFICIALES USANDO PRUEBAS DE TOXICIDAD. Eidenar 2013, 12, 79-90.

Bharagava RN, M. S. Hexavalent Chromium Reduction Potential of Cellulosimicrobium Sp. Isolated from Common Effluent Treatment Plant of Tannery Industries. Ecotoxicol. enviromental Saf. 2018, 147, 147:102-109.

Boffetta P. "Carcinogenety of Trace Elements with Reference to Evaluation Made by International Agency for Research on Cancer. Scandinavian Journal Working 
Environmental Health. Vol. 19, Supl. 1. Pgs 67-70. 1993.

Boran, M.; Altinok, I. A Review of Heavy Metals in Water, Sediment and Living Organisms in the Black Sea. Turkish J. Fish. Aquat. Sci. 2010, $10(4)$.

Ceren Haktanır, H. Önder Özbelge, N. B. \& L. Y. Removal of Hexavalent Chromium Anions via Polymer Enhanced Ultrafiltration Using a Fully Ionized Polyelectrolyte. Sep. Sci. Technol. 2017, 52, 2487-2497.

\section{CERYAK,S;ZINGARIELLO,C;O'BRIEN,T;}

PATIERNO,S.T.2004.Induction of proapoptotic and cell cycle-inhibiting genes in chromium (VI)-treated human lung fibroblasts: lack of effect of ERK, Mol. Cell. Biochem. 255:139-149

CHAVEZ PORRAS,A.2010.Descripción de la nocividad del cromo proveniente de la industria curtiembre y de las posibles formas de removerlo. Revista Ingenierías Universidad de Medellín. Colombia.9(17):4149.

Cuberos, E.; Rodriguez, A. I.; Prieto, E. Niveles de Cromo Y Alteraciones de Salud En Una Población Expuesta a Las Actividades de Curtiembres En Bogotá, Colombia. Rev. Salud Pública 2009, 11 (2), 278-289.

$\begin{array}{ccccrr}\text { Desastres, P. D. de } & \text { G. del } & \text { R. } & \text { de. } \\ \text { CARACTERIZACIÓN } & & & & \text { DEL } \\ \text { DEPARTAMENTO } & \text { NORTE } & & \text { DE } \\ \text { SANTANDER. 2014. } & & & & \end{array}$

Departamento Nacional de Planeación Fundación Corona. "Microempresa Y Competitividad, Cuero". CEINNOVA 5. Centro de Innovación Y Servicios Para La Industria Del Calzado. Impresión Panamericana. Bogotá. 1998.

De FLORA, S.2000.Threshold mechanisms and site specificity in chromium (VI) carcinogenesis. Carcinogenesis 21: 533-541.

Gil Tocados G, Manrique Plaza A, Fernández JM. "Introducción a La Dermatitis de Contacto
En La Construcción" Historia Del Cromo. Facultad de Medicina. Universidad de Cádiz. España. 2002.

G. P. S. Priebe, E. Kipper, A. L. Gusmão, N. R. Marcilio, M. G. Anaerobic Digestion of Chrome-Tanned Leather Waste for Biogas Production. J. Clean. Prod. 2016, 129, 410 416.

Herrero O, Servia MJ, P. R. Desarrollo de Una Estrategia Inteligente Para La Evaluación de La Salud de Ecositemas Acuáticos. Asoc. Española Toxicol. 2017, 34(2), 83-187.

Hernández Garcia Jessika Andrea ., Ariza Garcia Jherson, Cano Calle Herminsul de Jesús ,Contreras Pineda Jorge .2013.Estandarización de una técnica para la certificación de jardines clonales de plantas de cacao(Theobroma cacao ) usando marcadores moleculares rapd. Bistua:Revista de la Facultad de Ciencias Básicas.10(2):7584

Hexavalent Chromium Induces Testicular Dysfunction in Small Indian Mongoose (Herpestes Javanicus) Inhabiting Tanneries Area of Kasur District, Pakistan. J. Environ. Anal. Toxicol. 2017, 7 (5), 32.

HOLMES,A.L;WISE,S.S;WISE,J.P.2008.

Carcinogenicity of hexavalent chromium.Indian J. Med. Res. 128 (2008) 353-372.

JENNETTE,K.W.1982.Microsomal reduction of the carcinogen chromate produces chromium $(V)$. J. Am. Chem. Soc. 874.

K, S. Hexavalent Chromium and Bladder Cancer Risk. Actas Urol. españolas 2011.

Kristen, P.; Nickens, A.; Atierno, S.R.; Cceryak, S. 2010. Chromium genotoxicity: A doubleedged sword. Chemico-Biological Interactions.188:276-288

Linfeng Chen, Jing Zhang, Yaxian Zhu, Y. Z. Interaction of chromium(III) or chromium(VI) with Catalase and Its Effect on the Structure 
and Function of Catalase: An in Vitro Study. Food Chem. 2018, 244, 378-385.

Meléndez I., Pardo E., Quijano A. Actividad genotóxica de aguas antes y despues de clorar en la planta de potabilización empopamplona. Bistua:Revista de la Facultad de Ciencias Básicas.2015.13(2):1223.

Meneses, Alexander.;Hernández, Eudes E.2013.Identificaciòn de emisiones directas $e$ indirectas de GEI en el sector tratamiento $y$ disposición de aguas residuales:bases para la formulación de proyectos MDL en Ptar.Bistua.2(1):60-69

Ning Li, Yuan Kang, Weijian Pan, Lixuan Zeng, Qiuyun Zhang, J. L. Concentration and Transportation of Heavy Metals in Vegetables and Risk Assessment of Human Exposure to Bioaccessible Heavy Metals in Soil near a Waste-Incinerator Site, South China. Sci. Total Environ. 2015, 521-522, 144-151.

O'BRIEN,T;XU,J; PATIERNO,S.R.2001.Effects of glutathione on chromium-induced DNA crosslinking and DNA polymerase arrest, Mol. Cell. Biochem. 222:173-182.

O'BRIEN,T.J;CERYAK,S;PATIERNO,S.R. 2003.Complexities of chromium carcinogenesis:role of cellular response, repair and recovery mechanisms, Mutat. Res.533:3-36.

\section{O'BRIEN,T.J;} WITCHER,P; BROOKS,B;PATIERNO,S.R. 2009. DNA polymerase zeta is essential for hexavalent chromium-induced mutagenesis, Mutat. Res. $663: 77-83$.

OMS, 2003: heavy metals in drinking-water. Documento de referencia para la elaboración de las guías de la OMS para la calidad del agua potable. Ginebra (Suiza), Organización Mundial de la salud (WHO/SDE/WSH).

PANDRANGI,R;PETRAS,M;RALPH,S;VRZOC,M. 1995.Alkaline single cell gel (comet) ssay and genotoxicity monitoring using bulheads and carp.Environ.Mol.Mutagen.26(4):345-56

Prada, O. L. B. EVALUACIÓN Y REDUCCIÓN DE LOS NIVELES DE CROMO EN MUESTRAS DE AGUAS RESIDUALES PROVENIENTES DE CURTIEMBRES. L'esprit Ingénieux 2014, 5 N.1, 6-12.

PENAGOS, N. E. O. RECUPERACIÓN Y REUTILIZACIÓN DE CROMO DE LAS AGUAS RESIDUALES DEL PROCESO DE CURTIDO DE CURTIEMBRES DE SAN BENITO (BOGOTÁ), MEDIANTE UN PROCESO SOSTENIBLE Y VIABLE TECNOLÓGICAMENTE, 2013.

PERKIN ELMER. (1982). Analytical Methods for Atomic Absorption Spectrophotometry. Norwalk, Connecticut. U.S.A.

Quimipiel. "Manual Técnico Para La Ingeniería Del Cuero”. Editorial Igratal Ltda. Primera Edición. Bogotá.

Sayago, U. F. C. Design and Evaluation of a Biosystem Water Treatment Pilot-Scale Tannery through Eichhornia Crassipes. Rev. Colomb. Biotecnol. 2016, 18(2), 74-81.

SALNIKOW,K \& ZHITKOVICH, A.2008. Genetic and epigenetic mechanisms in metal carcinogenesis and cocarcinogenesis: nickel, arsenic, and chromium, Chem. Res.Toxicol. $21: 28-44$.

SINGH, N.P; McCOY, M.T ;TICE, R,R; SCHNEIDER, E.L.1998.A simple technique for quantitation of low levels of DNA damage in individual cells.Exp.Cell Res.75(1):184-91

SEDMAN,R.M;BEAUMONT,J;McDONALD,T.A;R EYNOLDS,S;KROWECH, G;HOWD,R. 2006. Review of the evidence regarding the carcinogenicity of hexavalent chromium in drinking water, J. Environ. Sci. Health. C. Environ. Carcinog.Ecotoxicol. Rev. 24:155182

SUGIYAMA, M.1991.Effects of vitamins on chromium(VI)-induced damage, Environ.Health Perspect. 92:63-70. 
SUGIYAMA,M;TSUZUKI,K;LIN,X;COSTA,M.199 2.Potentiation of sodium Chromate (VI)induced chromosomal aberrations and mutation by vitamin B2in Chinese hamster V79 cells, Mutat. Res. 283:211-214.

TAGLIARI, K.C.; CECCHINI, R.; ROCHA, J.A.V.; VARGAS, V.M.F. 2004. Mutagenicity of sediment and biomarkers of oxidative stress in fish from aquatic environments under the influence of tanneries. Mutat. Res. 561:101117.

Téllez J, Gutiérrez M, Patiño N. “Memorias Del Curso Taller Aspectos Toxicologicos de Plaguicidas Y Metales Pesados". Departamento de Toxicología. Universidad Nacional de Colombia. Bogotá, Julio de 2003.

VARGAS, V.; MIGLIAVACCA, S.; MELO, A.; HORN, R. 2001. Genotoxicity assessment in aquatic environments under the influence of heavy metals and organic contaminants. Mut. Res. 490:141-158.

Wang X, Z. S. Distribution Characteristics and Ecological Risk Assessment of Toxic Heavy Metals and Metalloid in Surface Water of Lakes in Daqing Heilongjiang Province, China. Ecotoxicology 2014, 23, 609-617.

Yenni Mirella Vásquez Perea, Jean Carlos Villamil Poveda, Ligia Consuelo Sánchez Leal, A. G. L. D. Evaluation of a System of Fixed Media Support for a Film Capable of Reducing Microbial Cr (VI) of Tannerysewage Sludge. Nova 2014, 12(21), 57-66.

Yulieth Reyes, Inés Vergara, Omar Torres, Mercedes Díaz-Lagos, E. E. G.-J. Contaminación Por Metales Pesados: Implicaciones En Salud, Ambiente $Y$ Seguridad Alimentaria. UPTC 2016, 16, N.2, 66-77.

Zahir Ur REHMAN, Sardar KHAN, Mohammad Tahir SHAH, Mark L BRUSSEAU, Said Akbar KHAN, J. M. Transfer of Heavy Metals from Soils to Vegetables and Associated Human Health Risk in Selected Sites in Pakistan. Pedosphere 2017. 